\title{
How pulse disturbances shape size-abundance pyramids
}

Claire Jacquet $1,2^{*}$, Isabelle Gounand 1,2 , Florian Altermatt 1,2

1 Department of Aquatic Ecology, Swiss Federal Institute of Aquatic Science and Technology, Eawag, Dübendorf, Switzerland

2 Department of Evolutionary Biology and Environmental Studies, University of Zurich, Zürich, Switzerland

* corresponding author: phone: +4158765 6726, fax: +41 587655802

Authors’ e-mail addresses: claire.jacquet@eawag.ch, isabelle.gounand@eawag.ch, florian.altermatt@eawag.ch

Statement of authorship: CJ and FA designed research, CJ conducted the experimental research, CJ and IG designed the theoretical research and IG did the mathematics and simulations to produce the theoretical figures. CJ wrote the first draft of the manuscript and all authors critically contributed to the edition of the paper.

Keywords: perturbations, extreme events, metabolic theory, body-size, community sizestructure, size spectrum, protist communities, disturbance frequency, disturbance intensity.

Running title: How pulse disturbances shape size-abundance pyramids.

Type of article: Letter.

Abstract: 144 words.

Main text: 4750 words.

References: 67.

The manuscript contains 6 Figures and Supporting Information.

This document is the accepted manuscript version of the following article:

Jacquet, C., Gounand, I., \& Altermatt, F. (2020). How pulse disturbances shape sizeabundance pyramids. Ecology Letters, 23(6), 1014-1023.

https://doi.org/10.1111/e1e.13508 


\section{Abstract}

2 Ecological pyramids represent the distribution of abundance and biomass of living organisms

3 across body-sizes. Our understanding of their expected shape relies on the assumption of invariant

4 steady-state conditions. However, most of the world's ecosystems experience disturbances that

5 keep them far from such a steady state. Here, using the allometric scaling between population

6 growth rate and body-size, we predict the response of size-abundance pyramids within a trophic

7 guild to any combination of disturbance frequency and intensity affecting all species in a similar

8 way. We show that disturbances narrow the base of size-abundance pyramids, lower their height

9 and decrease total community biomass in a nonlinear way. An experimental test using microbial

10 communities demonstrates that the model captures well the effect of disturbances on empirical

11 pyramids. Overall, we demonstrate both theoretically and experimentally how disturbances that are 12 not size-selective can nonetheless have disproportionate impacts on large species.

\section{INTRODUCTION}

15 Ecological pyramids, which represent the distribution of abundance and biomass of 16 organisms across body-sizes or trophic levels, reveal one of the most striking regularities among 17 communities (Elton 1927; Lindeman 1942; Trebilco et al. 2013). Several types of pyramids have 18 been reported in ecological research, as well as distinct underlying mechanisms to explain their 19 shape. For example, trophic pyramids describe the distribution of abundance or biomass along 20 discrete trophic levels (Fig. 1a). The inefficiency in energy transfer from resources to consumers

21 as well as strong self-regulation within trophic levels provide the main explanation for their shape

22 (Lindeman 1942; Barbier \& Loreau 2019). Alternatively, size-abundance pyramids (Fig. 1b,c), also

23 known as the pyramid of numbers (Elton 1927), the Damuth law (Damuth 1981), or the abundance

24 size spectrum (Sprules \& Barth 2016), describe the distribution of abundance across body-sizes 
25 and can be studied both within and across trophic guilds (Elton 1927; Trebilco et al. 2013). The

26 energetic equivalence rule, along with the metabolic theory of ecology, provide theoretical

27 expectations regarding the shape of such size-abundance pyramids: in a community where all

28 individuals feed on a common resource (i.e. within a trophic group), population abundance should

29 be proportional to $M^{-0.75}$, where $M$ is body-size, and biomass should be proportional to $M^{0.25}$

30 (Damuth 1981; Brown et al. 2004; White et al. 2007).

31 As with most concepts in ecology, these relationships correspond to theoretical baselines

32 that are predicted under steady-state conditions, which are rarely met in nature (DeAngelis \&

33 Waterhouse 1987; Hastings 2004, 2010). Most natural ecosystems and communities are exposed

34 to a wide range of environmental fluctuations and disturbances, ranging from harvesting to extreme

35 weather events. Furthermore, many of these disturbances are expected to increase in frequency and

36 intensity in the context of global change, as illustrated by recent large scale wildfires, floods or

37 hurricanes (Coumou \& Rahmstorf 2012; Hughes et al. 2017; Harris et al. 2018). Such disturbances

38 increase population mortality and could trigger even faster changes in community structure and

39 dynamics than gradual changes in average conditions (Jentsch et al. 2009; Wernberg et al. 2013;

40 Woodward et al. 2016).

$41 \quad$ Despite the extensive literature on disturbance ecology (Sousa 1984; Yodzis 1988; Petraitis

42 et al. 1989; Fox 2013; Dantas et al. 2016; Thom \& Seidl 2016), the effects of disturbances on

43 community structure and biomass distribution remain poorly understood (Donohue et al. 2016).

44 On the one hand, ecologists have often focused on the consequences of environmental disturbances

45 on species richness (Huston 1979; Haddad et al. 2008; Bongers et al. 2009) and the coexistence of

46 competing species (Violle et al. 2010; Miller et al. 2011; Fox 2013), rather than on body-size and

47 biomass distribution (but see Woodward et al. (2016)). As such, the specific identity of species 48 resistant (or not) to disturbances has received ample attention, with various definitions of 
49 disturbance-resistant species groups (Sousa 1980, 1984; Lavorel et al. 1997). These studies have

50 pointed out key demographic traits, notably population growth rate and carrying capacity, that

51 determine species' capacities to persist in a disturbed environment (McGill et al. 2006; Haddad et

52 al. 2008; Enquist et al. 2015; Woodward et al. 2016). On the other hand, the metabolic theory of

53 ecology uses the scaling of metabolic rate with body-size to predict a set of structural and functional

54 characteristics across biological scales (Brown et al. 2004). At the community level, it

55 demonstrates how size-abundance pyramids emerge from the scaling of population growth rate and

56 abundance with body-size (Trebilco et al. 2013). Surprisingly, a formal integration of the theory

57 on disturbances with the metabolic theory of ecology is still lacking, but would allow ecologists to

58 generalize and predict the effect of environmental disturbances on the shape of size-abundance

59 pyramids.

60 Here, we integrate these two disconnected fields by developing a size-based model for

61 population persistence, assuming that the scaling of population growth rate with body-size is the

62 leading mechanism determining the response of size-abundance pyramids to disturbances. We

63 predict the shape of size-abundance pyramids within a trophic guild in response to repeated pulse

64 disturbances of varying frequency and intensity affecting all species in a similar way, regardless of

65 their size. Such disturbances represent a wide range of environmental pressures that increase

66 species mortality, such as floods, wildfires, or hurricanes. They differ from the disturbance studies

67 developed in fishery sciences, that specifically addressed the effect of a press, size-selective

68 disturbance (i.e. fishing) on the abundance size spectrum (Jennings et al. 2002; Shin et al. 2005;

69 Petchey \& Belgrano 2010; Sprules \& Barth 2016). We then experimentally test the predicted

70 responses of size-abundance pyramids and standing biomass to disturbances, using microbial

71 communities composed of aquatic species with body-sizes and populations densities varying over 
72 several orders of magnitudes. We finally discuss the general implications of our findings for the

73 structure and functioning of communities exposed to environmental disturbances.

\section{MATERIALS AND METHODS}

\section{A model for size-abundance pyramids exposed to disturbances}

We build a mechanistic model to predict how disturbance frequency and intensity modulate

78 the shape of size-abundance pyramids and community total biomass. We describe the dynamics of

79 population abundance $N$ with a logistic model:

$$
\frac{d N}{d t}=r N\left(1-\frac{N}{K}\right)
$$

81 where $r$ is population growth rate and $K$ is population carrying capacity. We model a disturbance

82 regime, corresponding to a recurrent abundance reduction, of intensity $I$ (fraction of abundance)

83 and frequency $f$ or period $T=1 / f$ (time between two disturbances, Fig. $2 \mathrm{a}$ ). We can demonstrate that

84 a population persists in a disturbed environment only if its growth rate balances the long-term effect

85 of the disturbance regime (adapted from Harvey et al. 2016), that is:

$$
r>-\frac{\ln (1-I)}{T}
$$

From equation (2), we can predict the set of disturbance regimes a population can sustain

88 according to its growth rate (Fig. 2 b), as well as the minimum generation time $(1 / r)$ needed to

89 maintain a viable population (Fig. S1). We then use the allometric relationship between population

90 growth rate $r$ and average body-size $M$, that is $r=c \times M^{a}$ with $a=-1 / 4$ (Brown et al. 2004;

91 Savage et al. 2004) and $c$ a positive constant, to derive the following size-specific criterion for

92 population persistence under a disturbance regime:

$$
M \leq\left(\frac{\ln (1-I)}{T \times c}\right)^{-4}
$$


94 Equation (3) indicates that a species can persist in a disturbed environment only if its average body-

95 size is below a certain value. Note that this analytical criterion is applicable to any biological and

96 temporal scale. Indeed, the disturbance frequency and population growth rate are expressed with

97 the same time unit and can range from hours (e.g. fast-growing microbial organisms) to years (e.g.

98 slow-growing organisms such as large mammals). To investigate the effect of disturbances on the

99 shape of size-abundance pyramids, we derive the mean abundance at dynamical equilibrium $\bar{N}$ of

100 a population under a given disturbance regime (i.e. averaged over a time period, see Appendix 1

101 for detailed steps), that is:

102

$$
\bar{N}=K\left(\frac{\ln (1-I)}{T \times r}+1\right)
$$

103 where $K$ corresponds to the carrying capacity of the population, which also scales with body-size

104 on a logarithmic scale (Brown \& Gillooly 2003; Brown et al. 2004): $\ln (K)=a_{K} \ln (M)+b_{K}$,

105 where $a_{K}$ and $b_{K}$ are normalizing constants. We use this allometric relationship to express mean

106 abundance as a function of mean population body-size and finally obtain:

$$
\ln (\bar{N})=a_{K} \ln (M)+b_{K}+\ln \left(\frac{\ln (1-I)}{T e^{a_{r} \ln (M)+b_{r}}}+1\right)
$$

108 The formula is valid when the expression in parentheses in the right-hand term is positive, which

109 corresponds to the persistence criteria given in equations (2) and (3). We express population

110 biomass, $B$, as the product of mean abundance at dynamical equilibrium, $\bar{N}$, and the average

111 individual body-size in the population, $M$, that is $B=\bar{N} M$.

112 We extend this approach to multispecies assemblages composed of potentially hundreds of

113 co-occurring species with different body-sizes (see detailed method in Appendix 2 and Table S1

114 for parameter values). We assume that all species' populations follow a logistic growth and are

115 constrained by intraspecific competition only (an assumption relaxed in Appendix 3). From

116 equation (3) and (5), we expect that disturbances will decrease the maximum size observed in the 
117 community as well as total biomass. We use this analytical approach to explore how community

118 size-structure, a more tractable representation of abundance distribution across size-classes 119 compared to pyramids (Fig. 1b), and total community biomass will respond to a whole landscape 120 of disturbance frequencies and intensities (Fig. 3).

\section{Disturbance experiment on microbial communities}

123 We conducted an experiment in aquatic microcosms inoculated with 13 protist species and

124 a set of common freshwater bacteria as a food resource. The protist species cover a wide range of

125 body-sizes (from 10-103 $\mu \mathrm{m}$ ) and densities (10-105 individuals/ml, Giometto et al. 2013). General

126 lab procedures follow the protocols described in Altermatt et al. (2015), and build upon previous

127 work on pulse disturbance effects on diversity (Altermatt et al. 2011; Harvey et al. 2016) and 128 invasion dynamics (Mächler \& Altermatt 2012). Detailed microcosm description and set-up are

129 presented in Appendix 4. In short, we performed a factorial experiment in which we varied

130 disturbance frequency and intensity, resulting in a total of twenty different disturbance regimes.

131 Disturbance was achieved by boiling a subsampled fraction of the well-mixed community in a

132 microwave so that all species experience the same level of density reduction. All protists were

133 killed by the microwaving process. We let the medium cool down before putting it back into the

134 microcosm. We disturbed microcosms at five intensities: 10, 30, 50, 70 and $90 \%$ and at four

135 frequencies: $f=0.08,0.11,0.16$ and 0.33 , corresponding to a disturbance every $12,9,6$ and 3 days,

136 respectively. The experiment lasted for 21 days, or about 10-50 generations depending on species.

137 Each disturbance regime was replicated six times. To control for the intrinsic variability of 138 community size-structure, we cultured eight undisturbed microcosms under the same conditions.

139 We sampled $0.2 \mathrm{ml}$ of each microcosm daily to quantify individual body-sizes (i.e. cell area in $140 \mu \mathrm{m} 2$ ), protist abundances (individuals/ $\mu 1$ ) and total community biomass (i.e. total bioarea in 
$141 \mu \mathrm{m} 2 / \mu \mathrm{l}$ ) using a standardized video procedure (Altermatt et al. 2015; Pennekamp et al. 2017). We

142 binned the observed individuals into twelve size-classes ranging from 0 to $1.6 \times 10_{5} \mu \mathrm{m}_{2}$ in order to

143 get statistically comparable community size-structures. Mean protist abundance and its standard

144 deviation in each size-class were calculated over 21 time points and 6 replicates (total of 126

145 observations) for each treatment and over 21 time points and 8 replicates (total of 168 observations)

146 for the control communities. We performed Welch two sample t-tests of mean comparison

147 (treatment versus control) to determine which disturbance regime had a significant effect on 148 community size-structure and total community biomass (Table S2).

\section{Model parameterization}

151 We parameterized the model using the experimental data in order to test the capacity of the model 152 to predict the effect of a given disturbance regime on the size-structure of real communities. The 153 model required the following input parameters: the carrying capacities of each size-class as well as

154 the slope and the intercept of the allometric relationship between growth rate and body-size. We

155 took the average abundances of the undisturbed communities ( 8 controls) to the estimate carrying 156 capacities in each size-class. We fitted a logistic growth model to the recovery dynamics of each 157 size-class after one disturbance $(\mathrm{I}=90 \%)$ to obtain growth rate estimations. Specifically, we used 158 the data from the treatment $\{I=90 \%, f=0.08\}$ (i.e. highest intensity, lowest frequency) to estimate 159 the parameters of a logistic growth model over 12 time points using the function $n l s()$ of the stats 160 package in $\mathrm{R}$ ( $\mathrm{R}$ Core Team 2019). We determined the relationship between growth rate and body161 size in our experimental communities using the 13 time-series (covering 6 size-classes) that 162 displayed a logistic growth. We obtained the following allometric relationship: $\ln (r)=$ $163-0.37 \times \ln (M)+3.75\left(\mathrm{p}\right.$-value $\left.=0.005, \mathrm{R}_{2}=0.47\right)$. Using this parameterization, we produced 
164 theoretical predictions on the size-abundance pyramids expected in the experimental disturbance

165 regimes. We then quantitively compared these predictions with the size-abundance pyramids

166 observed in the experimental communities. We performed ordinary least-squares regressions to

167 characterize the relationship between observed and predicted log-transformed mean abundances 168 among size-classes for all the disturbance regimes.

170 RESULTS

\section{Model predictions}

172 We first explore the effects of increasing disturbance frequency (Fig. 3a, c). Infrequent

173 disturbances do not strongly affect community size-structure and only decrease the mean

174 abundance of the largest size-classes (Fig. 3a, $f=0.1$ in dark blue). Maximum body-size gradually

175 decreases as disturbance frequency increases, corresponding to the extinction of large, slow-

176 growing species (Fig. 3a, $f=0.25$ in light blue). Disturbance frequency also affects the community

177 size-structure through its effect on mean abundance. For frequent disturbance events, the mean

178 abundance of all size-class decreases (Fig. $3 \mathrm{a}, f=0.5$ and 1 in orange and red respectively). The

179 effect of disturbance frequency on community-size structure have direct consequences for

180 community-level properties: we indeed observe an approximately linear decrease in total

181 community biomass $(\log )$ along a gradient of disturbance frequency, followed by an abrupt collapse

182 of the community for extreme disturbance regimes (Fig. 3c).

183 We then investigate the effect of increasing disturbance intensity (Fig. 3b, d). Similarly,

184 low intensity disturbances marginally affect community size-structure (Fig. 3c, $I=30 \%$ in blue)

185 and increasing disturbance intensity decreases maximum body-size and population mean

186 abundance. (Fig. 3b). Interestingly, the effect of disturbance intensity on community total biomass

187 is clearly nonlinear (Fig. 3d). Low to intermediate disturbance intensities do not affect total biomass 
188 when disturbance frequency is low (e.g. $f=0.1$ or 0.25 in Fig. 3d). However, strong intensities

189 affect all population abundances and trigger a sharp decrease in total biomass, culminating in a 190 crash of the system (e.g. $\{I>90 \%, f=0.25\}$ in Fig $3 \mathrm{~d}$ ).

\section{Experimental results}

We experimentally investigated the effect of disturbance frequency and intensity on the

194 size-structure of microbial communities. For a fixed intensity (set to $I=90 \%$ in Fig. 4a, see Fig.

195 S2 for other intensities), infrequent disturbances (i.e. $f=0.08$ and $f=0.11$ ) had a significant

196 negative impact only on the mean abundance of intermediate size-classes (between $\exp (9.6)$ and

$197 \exp (10.5) \mu \mathrm{m} 2$, Welch two sample t-tests: $\mathrm{t} \geq 2.6$, $\mathrm{p}$-values $\leq 0.02$, Table $\mathrm{S} 2$ ). When disturbance

198 frequency increased to $f=0.16$, the mean abundance of the smallest size-class also decreased $(\mathrm{t}=$

1993.6 , p-value $=0.01$, Table S2). Finally, at even more frequent disturbances $(f=0.33)$, all size-

200 classes were negatively impacted, except the smallest one (Fig 5a and Table S3). Overall,

201 increasing disturbance frequency led to an abundance depletion at intermediate sizes compared to

202 undisturbed control communities.

203 Similarly, for a fixed frequency (set to $f=0.33$ in Fig. 4b, see Fig. S3 for other frequencies),

204 a low disturbance intensity $I=10 \%$ (Fig. 4b) only affected intermediate size-classes (between

$205 \exp (10)$ and $\exp (10.5) \mu \mathrm{m} 2, \mathrm{t} \geq 4.5, \mathrm{p}$-values $\leq 0.001$, Table S2). Disturbance intensities $I=30 \%$

206 and 50\% had a negative effect on the mean abundance of larger size-classes (between $\exp (10)$ and

$207 \exp (11) \mu \mathrm{m} 2, \mathrm{t} \geq 2.8, \mathrm{p}$-values $\leq 0.03$, Table S2). Finally, intensities $I=70 \%$ and $I=90 \%$ had an

208 impact on all size-classes, except the smallest size-class that were not negatively impacted by

209 change in disturbance intensity (Fig. 4b, Table S2). Interestingly, the following disturbance

210 regimes had a positive effect of on the mean abundance of the smallest size-class: $\{I=30 \%, f=$ 
$2110.33\}, \mathrm{t}=-6.1$, p-value $<0.001$, (Fig. 4b), as well as $\{I=50 \%, f=0.16\}$ and $\{I=70 \%, f=0.11\}$

212 (Table S2, Fig. S2 and S3).

213 At the community-level, total biomass gradually decreased with disturbance frequency as

214 expected by theory (Fig. 4c). All frequencies had a significant negative effect on total biomass

215 compared to controls ( $\mathrm{t} \geq 8, \mathrm{p}$-value $<0.001$, Fig 4c). Disturbance intensities $I=10 \%$ and $30 \%$ had

216 no significant effects on total community biomass $\left(I_{10 \%}: \mathrm{t}=0.75\right.$, $\mathrm{p}$-value $=0.48, I_{30 \%}: \mathrm{t}=0.5$, $\mathrm{p}$ -

217 value $=0.63)$, while total biomass strongly decreased for intensities above $I=50 \%(I 50 \%: \mathrm{t}=6.1$,

218 p-value $<0.001, I 70 \%: \mathrm{t}=12.7, \mathrm{p}$-value $<0.001, I 90 \%: \mathrm{t}=14.2$, p-value $<0.001$, Fig. 4d).

\section{Observed versus predicted effect of disturbances on size-abundance pyramids}

We then compared our experimental results with the predictions of the model parameterized

222 for our freshwater microbial communities (Figure 5). The model predicted well the observed mean

223 abundances relative to carrying capacity for all the disturbance regimes in most of the size-classes.

224 The slope of the linear regression between observed and predicted log mean abundances, including

225 all size-classes in all disturbance regimes (240 points), was very close to the 1:1 line, which

226 indicates a very good fit (Figure 5a, linear regression: $y=-0.012+1.01 x, R_{2}=0.96$, $p$-value $<$

227 0.001). Additionally, the intercept of the linear regression was not significantly different from zero

$228(\mathrm{t}=0.95, \mathrm{p}$-value $=0.34)$. We illustrate in Figure $5 \mathrm{~b}-\mathrm{d}$ the similarities as well as the differences

229 between the predicted and observed community size-structures for varying disturbance frequencies

230 with a disturbance intensity fixed to $I=90 \%$ (other disturbance regimes are shown in Figs. S4-S5).

231 Overall, the predicted community structures were very similar to the observed ones. The model,

232 however, often underestimated the mean abundance in the smallest size-class (Figure 5d).

233 Furthermore, as mentioned in the previous section, some disturbance regimes had a positive effect 
234 of on the mean abundance of the smallest size-class, which cannot, by construction, be predicted

235 by our model. We discuss below how this pattern can be explained by a disruption of biotic

236 interactions following a disturbance and present further analyses using a predator-prey model to

237 support this possible explanation (Fig. 6c, Appendix 3).

239 DISCUSSION

240 Most theories in community ecology have been developed under the assumption of steady-

241 state conditions (Hastings 2010). Yet, most of the world's ecosystems - specifically $\geq 75 \%$ of

242 land/freshwater and $50 \%$ of marine systems - have been altered by human activities and are facing

243 disturbances that put them clearly outside of such a steady state (IPBES 2018). Thus, to meet the

244 societal demand for an ecological science able to predict how ecosystems will respond to global 245 change (Petchey et al. 2015; Urban et al. 2016), this assumption needs to be relaxed. The challenge

246 is to develop models that make quantitative predictions regarding the impact of fluctuating

247 environmental conditions on the structural and functional characteristics of biological systems.

249 Consequences of the growth-size relationship for communities exposed to disturbances

250 Here, we provide a robust and simple approach for predicting the size-structure of 251 communities exposed to any combination of disturbance frequency and intensity affecting all 252 species in a similar way, regardless of their body-size. We combine theory on disturbances with 253 the metabolic theory of ecology and assume that the scaling of population growth rate with body254 size is the leading mechanism determining the response of size-abundance pyramids to 255 disturbances. The model makes an important advance over the steady-state predictions of the 256 metabolic theory of ecology as it links quantitatively the shape of a size-abundance pyramid to the 257 disturbance regime experienced by the community (Fig. 6a-b). Overall, increasing disturbance 
258 frequency or intensity narrows the bases of size-abundance pyramids and lowers their height. This

259 corresponds to the extinction of the largest species and a general reduction of population mean

260 abundances in all size-classes. Hence, we demonstrate that disturbances that are not size-selective

261 and do not target large species have nonetheless a higher impact on large species than on smaller 262 ones.

263 The model is applicable across all biological and temporal scales as population growth rate 264 and disturbance frequency are expressed with the same time units. Equation (2) can also apply to 265 populations that do not show a scaling relationship between growth rate and body-size and predicts 266 which disturbance regimes a species can sustain, or not, based on its generation time (Figs. 2 and 267 S1). Importantly, our results are not specific to repeated pulse disturbances but also hold for press 268 disturbances, which will affect the shape of size-abundance pyramids in an equivalent way (see 269 Appendix 1 for a mathematical demonstration).

270 Our model offers a new perspective on community responses to disturbances by exploring 271 the effect of repeated pulse disturbances of varying frequency and intensity on community size 272 structure. The majority of theoretical studies on community stability have focused on local stability, 273 which examine community's response to small pulse disturbances around one single equilibrium 274 (Donohue et al. 2016), reflecting the great interest for the so-called diversity-stability debate (May 275 1972; McCann 2000; Allesina \& Tang 2012; Jacquet et al. 2016). Our approach goes beyond local 276 stability measures at the vicinity of one single attractor and is applicable to any combination of 277 disturbance frequency or intensity. It predicts which species, based on its growth rate, can persist 278 or not and how the abundances of the remaining species will be affected by a whole gradient of 279 disturbances.

280 Note that the model depends on a number of technical assumptions. First, we restricted our 281 theoretical approach to disturbance regimes where pulse disturbances are applied at fixed intervals 
282 with a fixed intensity. This choice, though relatively simplistic, allowed us to mirror the disturbance

283 regimes applied to the experimental communities. To generalize, we also performed simulations

284 where we added stochasticity in the frequency and intensity of the disturbance regime to test the

285 sensitivity of the theoretical results to variability in the periodicity and intensity of disturbances

286 (Appendix 2). Our results were qualitatively robust to the addition of noise around average values

287 of disturbance frequency and intensity, which simply increased the negative effect of one given

288 disturbance regime on the largest size-classes (Fig. S6). Second, we consider that the allometric

289 parameters of the relationships between population growth rate, carrying capacity and body-size

290 are the same for all species (i.e. same slopes and intercepts). We therefore performed sensitivity

291 analyses of Equation (5) and demonstrate that our results are robust to variation in these allometric

292 parameters (Appendix 2, Fig. S7-8).

\section{Experimental test of the theory}

The disturbance experiment on microbial communities showed some similarities but also model, total community biomass gradually decreased with disturbance frequency and in a more nonlinear way with disturbance intensity (Fig. 4c-d, and Fig. 3c-d for the theoretical predictions). Interestingly, it was the intermediate and not the largest size-classes that were the most sensitive

300 to disturbances in the microbial community. We provide below two possible explanations for this 301 observation. Most likely, the abundances of the largest size-class might be already too low, and

302 therefore too close to the methodologically-defined detection threshold, in the control communities 303 to observe a significant effect of the disturbances of these size-classes. Second, this might be 304 explained by the duration of the experiment (21 days), which was not long enough to capture the 305 extinction of the largest species. We estimated the time to reach the dynamical equilibrium in the 
experiment with the model parameterized with experimental data (see Table S3). The model predicted that equilibrium is reached by the end of the experiment (21 days) for the size-classes considered in all disturbance regimes but the strongest. With the highest frequency and intensity $\{I=90 \% ; f=0.33\}$ the equilibrium is reached by the three smallest size-classes (in 12, 18, and 21 days respectively).

Additionally, some combinations of disturbance frequency and intensity had a positive

312 effect on the smallest size-class of microbes compared to controls, which corresponded to the main

313 departure from the theoretical predictions (Figure 4a-b and Figure 5d). This could be explained by

314 a disruption of biotic interactions (predation or competition) following a disturbance, allowing the

315 remaining small species to grow in higher densities in the absence of other species (Cox \& Ricklefs

316 1977; Ritchie \& Johnson 2009; Bolnick et al. 2010). Such "interaction-release" mechanism could

317 not be captured by our model of co-occurring species. We discuss below how interspecific 318 interactions, such as competition, predation or parasitism, could modulate the shape of size-

319 abundance pyramids exposed to disturbances.

321 Extending the model to communities of interacting species

To observe an "interaction-release" effect that will widen the pyramid's base, two

323 conditions are required (but not sufficient): (i) the existence of a significant mismatch between the

324 growth rates of the two interacting species, leading to differential response to disturbances, and (ii)

325 the species with the slowest growth rate has a negative effect on the other species (i.e. predator,

326 competitor or parasite). The latter condition seems unlikely for parasitism. For competitive

327 interactions, a "competition-release" effect can potentially increase the abundance of small, fast-

328 growing species that will recover faster from a disturbance event compared to larger competitors

329 (e.g. Xi et al. (2019)). Finally, the existence of a "predation-release" effect is very likely as 
330 predators are generally larger than their prey and have slower growth rates (Brose et al. 2006, 2016;

331 Barnes et al. 2010). In an additional analysis, we performed simulations using a predator-prey

332 model to explore in which conditions a "predation-release" effect could increase the abundance of

333 small prey species (see Appendix 3 for detailed methods). We found that small to intermediate

334 disturbance regimes can increase average prey abundance through a "predation-release" effect,

335 which should generate size-abundance pyramids with a wider base (Fig. 6c). This effect vanishes

336 above some disturbance thresholds, where prey species are also negatively impacted by

337 disturbances (Fig. 6c and Figs. S9-S11).

338 Our model cannot capture cascading effects triggered by complex interactions networks in

339 its current form. A promising future direction is the extension of the model to multitrophic

340 communities, which will allow further explorations of the potential of interspecific interactions to

341 modulate the impact of disturbances on size-abundance pyramids and community biomass. Indeed,

342 it is likely that predator species will also be impacted indirectly through a bottom-up transmission

343 of the disturbances (i.e. decrease in prey availability).

\section{Additional mechanisms shaping size-abundance pyramids exposed to disturbances}

346 Here, we propose a systematic approach, based on the metabolic theory of ecology, to

347 predict the response of size-abundance pyramids to persistent disturbances. Our results are specific

348 to a class of persistent disturbances (i.e. pulse or press) that affect the abundance of all species in

349 a similar way, regardless of their specific body-size or growth rate. We also assume that the leading

350 mechanism that determines the response of size-abundance pyramids to this type of disturbances

351 is the allometric relationship between species growth rate and body-size. However, additional

352 mechanisms can generate size-dependent abundances or size-dependent responses to disturbances

353 in real world ecosystems. First, species sensitivity to disturbances that are not size-selective can be 
354 nonetheless unequal among size classes, with particular size-classes being more resistant to a given

355 disturbance intensity. For example, strong windstorms or droughts generally cause greater 356 mortality among larger or taller trees (Woods 2004; Hurst et al. 2011; Bennett et al. 2015). Second,

357 from a spatial perspective, size-specific mobility and immigration-extinction dynamics could 358 largely affect the relationship between species recovery dynamics and their size (McCann et al.

359 2005; Jacquet et al. 2017). It would be interesting to extend our approach to metacommunities,

360 where the depletion of large species in a disturbed habitat patch could be balanced by immigration

361 from undisturbed neighboring patches (Pawar 2015).

362 Finally, some disturbances can be size-selective, as illustrated by studies on abundance size

363 spectra that specifically addressed the effect of a press, size-selective disturbance, often reflecting

364 disturbances expected under commercial fishing (Shin et al. 2005; Sprules \& Barth 2016). Our

365 model can easily be refined to more specific cases, in which disturbances have unequal effects on

366 species, by adding size-specific disturbance intensities to the model. The abundance size spectra

367 of harvested fish communities are generally characterized by steeper slopes than unfished

368 communities, and are used as a size-based indicator of fisheries exploitation (Shin et al. 2005;

369 Petchey \& Belgrano 2010; Sprules \& Barth 2016). We demonstrate that size-abundance pyramids

370 are also predictably affected by more general pulse disturbances that are not size-selective such as

371 floods or wildfires. Hence, when compared to a reference state, size-abundance pyramids provide

372 information on the level of disturbances an ecosystem is facing and could be used as "universal

373 indicators of ecological status", as advocated in Petchey \& Belgrano (2010).

\section{Conclusion}

376 Our findings have direct implications regarding the effects of disturbances on ecosystem

377 functioning. Indeed, the model makes predictions on total biomass and demographic traits 
378 correlated to productivity rate and energy flows, which are among the most relevant metrics to

379 quantify ecosystem functioning (Oliver et al. 2015; Schramski et al. 2015; Brose et al. 2016;

380 Barnes et al. 2018). In the current context of global change, we demonstrate that the expected

381 increase in disturbance frequency and intensity should accelerate the extinction of the largest

382 species, leading to an increasing proportion of communities dominated by small, fast-growing

383 species and lower levels of standing biomass. Importantly, the effect of increasing disturbance

384 regimes will be nonlinear and abrupt changes in community structure and functioning are expected

385 once a disturbance threshold affecting the equilibrium abundances of smaller species is reached.

387 DATA AVAILABILITY STATEMENT

388 The data supporting the experimental results as well as a Rmarkdown document, which explains

389 in detail the theoretical approach and produces the figures, are archived in the Dryad Digital

390 Repository: https://doi.org/10.5061/dryad.95x69p8g7.

391

\section{ACKNOWLEDGEMENT}

393 We thank Sereina Gut, Samuel Hürlemann and Silvana Käser for help during the laboratory work

394 and Chelsea J. Little for comments on the manuscript. We also thank Jean François Arnoldi,

395 Samraat Pawar and two anonymous reviewers for their helpful comments on previous versions of 396 the manuscript. Funding is from the Swiss National Science Foundation Grants No

397 PP00P3_179089, the University of Zurich Research Priority Program "URPP Global Change and

398 Biodiversity" (both to F.A.) and the University of Zurich Forschungskredit (to C.J. and I.G.).

400 REFERENCES

401 Allesina, S. \& Tang, S. (2012). Stability criteria for complex ecosystems. Nature, 483, 205-208. 
Altermatt, F., Fronhofer, E.A., Garnier, A., Giometto, A., Hammes, F., Klecka, J., et al. (2015). Big answers from small worlds: a user's guide for protist microcosms as a model system in ecology and evolution. Methods Ecol. Evol., 6, 218-231.

Altermatt, F., Schreiber, S. \& Holyoak, M. (2011). Interactive effects of disturbance and dispersal directionality on species richness and composition in metacommunities. Ecology, 92, 859870.

Barbier, M. \& Loreau, M. (2019). Pyramids and cascades: a synthesis of food chain functioning and stability. Ecol. Lett., 22, 405-419.

Barnes, A.D., Jochum, M., Lefcheck, J.S., Eisenhauer, N., Scherber, C., O’Connor, M.I., et al. (2018). Energy Flux: The Link between Multitrophic Biodiversity and Ecosystem Functioning. Trends Ecol. Evol., 33, 186-197.

Barnes, C., Maxwell, D., Reuman, D.C. \& Jennings, S. (2010). Global patterns in predator prey size relationships reveal size dependency of trophic transfer efficiency. Ecology, 91, $222-232$.

Bennett, A.C., McDowell, N.G., Allen, C.D. \& Anderson-Teixeira, K.J. (2015). Larger trees suffer most during drought in forests worldwide. Nat. Plants, 1, 15139.

Bolnick, D.I., Ingram, T., Stutz, W.E., Snowberg, L.K., Lau, O.L. \& Paull, J.S. (2010). Ecological release from interspecific competition leads to decoupled changes in population and individual niche width. Proc. R. Soc. B Biol. Sci., 277, 1789-1797.

Bongers, F., Poorter, L., Hawthorne, W.D. \& Sheil, D. (2009). The intermediate disturbance hypothesis applies to tropical forests, but disturbance contributes little to tree diversity. Ecol. Lett., 12, 798-805.

Brose, U., Blanchard, J.L., Eklöf, A., Galiana, N., Hartvig, M., R. Hirt, M., et al. (2016). Predicting the consequences of species loss using size-structured biodiversity approaches. 
Jacquet et al.

Biol. Rev., 49, n/a-n/a.

427

428

429

430

431

432

433

434

435

436

437

438

439

440

441

442

443

444

445

446

447

448

449

Brose, U., Jonsson, T. \& Berlow, E.L. (2006). Consumer-resource body size relationships in natural food webs. Ecology, 87, 2411-2417.

Brown, J.H. \& Gillooly, J.F. (2003). Ecological food webs : High-quality data facilitate theoretical unification. Proc. Natl. Acad. Sci., 100, 1467-1468.

Brown, J.H., Gillooly, J.F., Allen, A.P. \& Savage, V.M. (2004). Toward a metabolic theory of ecology. Ecology, 85, 1771-1789.

Coumou, D. \& Rahmstorf, S. (2012). A decade of weather extremes. Nat. Clim. Chang., 2, 491496.

Cox, G.W. \& Ricklefs, R.E. (1977). Species Diversity and Ecological Release in Caribbean Land Bird Faunas. Oikos, 28, 113.

Damuth, J. (1981). Population density and body size in mammals. Nature, 290, 699-700.

Dantas, V. de L., Hirota, M., Oliveira, R.S. \& Pausas, J.G. (2016). Disturbance maintains alternative biome states. Ecol. Lett., 19, 12-19.

DeAngelis, D.L. \& Waterhouse, J.C. (1987). Equilibrium and Nonequilibrium Concepts in Ecological Models. Ecol. Monogr., 57, 1-21.

Donohue, I., Hillebrand, H., Montoya, J.M., Petchey, O.L., Pimm, S.L., Fowler, M.S., et al. (2016). Navigating the complexity of ecological stability. Ecol. Lett., 19, 1172-1185.

Elton, C. (1927). Animal Ecology. Macmillan.

Enquist, B.J., Norberg, J., Bonser, S.P., Violle, C., Webb, C.T., Henderson, A., et al. (2015). Scaling from Traits to Ecosystems: Developing a General Trait Driver Theory via Integrating Trait-Based and Metabolic Scaling Theories. Adv. Ecol. Res., 52, 249-318.

Fox, J.W. (2013). The intermediate disturbance hypothesis should be abandoned. Trends Ecol. Evol., 28, 86-92. 
450 Giometto, A., Altermatt, F., Carrara, F., Maritan, A. \& Rinaldo, A. (2013). Scaling body size 451 fluctuations. Proc. Natl. Acad. Sci., 110, 4646-4650.

452 Haddad, N.M., Holyoak, M., Mata, T.M., Davies, K.F., Melbourne, B.A. \& Preston, K. (2008). 453 Species' traits predict the effects of disturbance and productivity on diversity. Ecol. Lett., $454 \quad 11,348-356$.

455 Harris, R.M.B., Beaumont, L.J., Vance, T.R., Tozer, C.R., Remenyi, T.A., Perkins-Kirkpatrick, 456 S.E., et al. (2018). Biological responses to the press and pulse of climate trends and extreme events. Nat. Clim. Chang., 8, 579-587.

458 Harvey, E., Gounand, I., Ganesanandamoorthy, P. \& Altermatt, F. (2016). Spatially cascading 459 effect of perturbations in experimental meta-ecosystems. Proc. R. Soc. B Biol. Sci., 283, $460 \quad 20161496$.

461 Hastings, A. (2004). Transients: the key to long-term ecological understanding? Trends Ecol. Evol., 19, 39-45.

463 Hastings, A. (2010). Timescales, dynamics, and ecological understanding. Ecology, 91, 34714643480.

465 Hughes, T.P., Kerry, J.T., Álvarez-Noriega, M., Álvarez-Romero, J.G., Anderson, K.D., Baird, 466 A.H., et al. (2017). Global warming and recurrent mass bleaching of corals. Nature, 543, $467 \quad 373-377$.

468 Hurst, J.M., Allen, R.B., Coomes, D.A. \& Duncan, R.P. (2011). Size-Specific Tree Mortality 469 Varies with Neighbourhood Crowding and Disturbance in a Montane Nothofagus Forest. $470 \quad$ PLoS One, 6, e26670.

471 Huston, M. (1979). A General Hypothesis of Species Diversity. Am. Nat., 113, 81-101.

472 IPBES. (2018). Summary for policymakers of the global assessment report on biodiversity and 473 ecosystem services of the Intergovernmental Science-Policy Platform on Biodiversity and 
Ecosystem Services.

475 Jacquet, C., Moritz, C., Morissette, L., Legagneux, P., Massol, F., Archambault, P., et al. (2016). 476 No complexity-stability relationship in empirical ecosystems. Nat. Commun., 7, 12573.

477 Jacquet, C., Mouillot, D., Kulbicki, M. \& Gravel, D. (2017). Extensions of Island Biogeography 478 Theory predict the scaling of functional trait composition with habitat area and isolation. $479 \quad$ Ecol. Lett., 20, 135-146.

480 Jennings, S., Warr, K.J. \& Mackinson, S. (2002). Use of size-based production and stable isotope 481 analyses to predict trophic transfer efficiencies and predator-prey body mass ratios in food 482 webs. Mar. Ecol. Prog. Ser., 240, 11-20.

483 Jentsch, A., Kreyling, J., Boettcher-Treschkow, J. \& Beierkuhnlein, C. (2009). Beyond gradual 484 warming: Extreme weather events alter flower phenology of European grassland and heath 485 species. Glob. Chang. Biol., 15, 837-849.

486 Lavorel, S., McIntyre, S., Landsberg, J. \& Forbes, T.D.A. (1997). Plant functional classifications: 487 from general groups to specific groups based on response to disturbance. Trends Ecol. Evol., $488 \quad 12,474-478$.

489 Lindeman, R. (1942). The trophic-dynamic aspect of ecology. Ecology, 23, 399-417.

490 Mächler, E. \& Altermatt, F. (2012). Interaction of Species Traits and Environmental Disturbance $491 \quad$ Predicts Invasion Success of Aquatic Microorganisms. PLoS One, 7.

492 May, R.M. (1972). Will a large complex system be stable? Nature, 238, 413-4.

493 McCann, K.S. (2000). The diversity-stability debate. Nature, 405, 228-233.

494 McCann, K.S., Rasmussen, J.B. \& Umbanhowar, J. (2005). The dynamics of spatially coupled 495 food webs. Ecol. Lett., 8, 513-23.

496 McGill, B., Enquist, B.J., Weiher, E. \& Westoby, M. (2006). Rebuilding community ecology 497 from functional traits. Trends Ecol. Evol., 21, 178-85. 
498 Miller, A.D., Roxburgh, S.H. \& Shea, K. (2011). How frequency and intensity shape diversity499 disturbance relationships. Proc. Natl. Acad. Sci., 108, 5643-5648.

500 Oliver, T.H., Heard, M.S., Isaac, N.J.B., Roy, D.B., Procter, D., Eigenbrod, F., et al. (2015).

501 Biodiversity and Resilience of Ecosystem Functions. Trends Ecol. Evol., 30, 673-684.

502 Pawar, S. (2015). The Role of Body Size Variation in Community Assembly. Trait. Ecol. - From

$503 \quad$ Struct. to Funct. 1st edn. Elsevier Ltd.

504 Petchey, O.L. \& Belgrano, A. (2010). Body-size distributions and size-spectra: universal

505 indicators of ecological status? Biol. Lett., 6, 434-437.

506 Petchey, O.L., Pontarp, M., Massie, T.M., Kéfi, S., Ozgul, A., Weilenmann, M., et al. (2015).

507 The ecological forecast horizon, and examples of its uses and determinants. Ecol. Lett., 18, $508 \quad 597-611$.

509 Petraitis, P.S., Latham, R.E. \& Niesenbaum, R.A. (1989). The Maintenance of Species Diversity

$510 \quad$ by Disturbance. Q. Rev. Biol., 64, 393-418.

511 R Core Team (2019). R: A language and environment for statistical computing. R Foundation for $512 \quad$ Statistical Computing, Vienna, Austria.

513 Ritchie, E.G. \& Johnson, C.N. (2009). Predator interactions, mesopredator release and 514 biodiversity conservation. Ecol. Lett., 12, 982-998.

515 Savage, V.M., Gillooly, J.F., Brown, J.H. \& Charnov, E.L. (2004). Effects of body size and 516 temperature on population growth. Am. Nat., 163, 429-41.

517 Schramski, J.R., Dell, A.I., Grady, J.M., Sibly, R.M. \& Brown, J.H. (2015). Metabolic theory

518 predicts whole-ecosystem properties. Proc. Natl. Acad. Sci., 112, 2617-2622.

519 Shin, Y.-J., Rochet, M.-J., Jennings, S., Field, J.G. \& Gislason, H. (2005). Using size-based 520 indicators to evaluate the ecosystem effects of fishing. ICES J. Mar. Sci., 62, 384-396.

521 Sousa, W.P. (1980). The responses of a community to disturbance: the importance of 
successional age and species' life histories. Oecologia, 45, 72-81.

523 Sousa, W.P. (1984). The Role of Disturbance in Natural Communities. Annu. Rev. Ecol. Syst., 15, $524 \quad 353-391$.

525 Sprules, W.G. \& Barth, L.E. (2016). Surfing the biomass size spectrum: Some remarks on 526 history, theory, and application. Can. J. Fish. Aquat. Sci., 73, 477-495.

527 Thom, D. \& Seidl, R. (2016). Natural disturbance impacts on ecosystem services and biodiversity 528 in temperate and boreal forests. Biol. Rev. Camb. Philos. Soc., 91, 760-781.

529 Trebilco, R., Baum, J.K., Salomon, A.K. \& Dulvy, N.K. (2013). Ecosystem ecology: size-based 530 constraints on the pyramids of life. Trends Ecol. Evol., 28, 423-431.

531 Urban, M.C., Bocedi, G., Hendry, A.P., Mihoub, J.-B., Peer, G., Singer, A., et al. (2016).

532 Improving the forecast for biodiversity under climate change. Science (80-. )., 353, $533 \quad$ aad8466-aad8466.

534 Violle, C., Pu, Z. \& Jiang, L. (2010). Experimental demonstration of the importance of 535 competition under disturbance. Proc. Natl. Acad. Sci., 107, 12925-12929.

536 Wernberg, T., Smale, D.A., Tuya, F., Thomsen, M.S., Langlois, T.J., De Bettignies, T., et al.

537 (2013). An extreme climatic event alters marine ecosystem structure in a global biodiversity 538 hotspot. Nat. Clim. Chang., 3, 78-82.

539 White, E.P., Ernest, S.K.M., Kerkhoff, A.J. \& Environnement, E.T. (2007). Relationships 540 between body size and abundance in ecology. Trends Ecol. Evol., 22, 323-30.

541 Woods, K.D. (2004). Intermediate disturbance in a late-successional hemlock-northern hardwood 542 forest. J. Ecol., 92, 464-476.

543 Woodward, G., Bonada, N., Brown, L.E., Death, R.G., Durance, I., Gray, C., et al. (2016). The 544 effects of climatic fluctuations and extreme events on running water ecosystems. Philos. 545 Trans. R. Soc., 371, 20150274. 
546 Xi, W., Peet, R.K., Lee, M.T. \& Urban, D.L. (2019). Hurricane disturbances, tree diversity, and

547 succession in North Carolina Piedmont forests, USA. J. For. Res., 30, 219-231.

548 Yodzis, P. (1988). The Indeterminacy of Ecological Interactions as Perceived Through

$549 \quad$ Perturbation Experiments. Ecology, 69, 508-515.

550 


\section{FIGURE LEGENDS}

552 Figure 1: A trophic pyramid (a) describes the distribution of biomass along discrete trophic levels,

553 and assumes that all species within a trophic level have the same functional traits. The community

554 size-structure (b) and the size-abundance pyramid (c) are equivalent size-centric representations of 555 ecological communities and are the focus of this study. They describe the distribution of abundance 556 across body-sizes and can be studied both within and across trophic levels. b) the community size-

557 structure depicts $\log ($ body-size $)$ on the $\mathrm{x}$-axis and $\log ($ abundance $)$ on the $y$-axis, while c) the size558 abundance pyramid shows $\log ($ abundance $)$ on the $\mathrm{x}$-axis and $\log$ (body-size) on the $\mathrm{y}$-axis. Note

559 that the area $A$ is the same in both panels. We use the community size-structure representation 560 throughout the paper as it facilitates comparisons between theory and experimental data, but see

561 Fig. 6 for a synthesis of our findings using the pyramid representation.

563 Figure 2: Population dynamics and persistence according to disturbance regime. a) Temporal 564 dynamics of two species experiencing the same disturbance regime. Species 1 has a smaller body565 size and therefore a higher growth rate than species 2. A population can persist only if its growth 566 rate balances the long-term effect of the disturbance regime. We derive in equation (4) the mean 567 abundance at dynamical equilibrium (i.e. temporal mean) of the persisting species experiencing 568 varying disturbance regimes. b) Isoclines of the persistence criterion in the disturbance regime 569 landscape according to population growth rate (numbers): on and above the line, the population of 570 a given growth rate goes extinct. Lines with the same color code as in panel (a) correspond to the 571 same growth rate.

573 Figure 3: Effects of disturbance frequency and intensity on community size-structure and average 574 total biomass at dynamical steady state. Analytical results derived from Equation (5). a) Effect of 
575 disturbance frequency (disturbance intensity is fixed to $50 \%$ abundance reduction), and b)

576 disturbance intensity (disturbance frequency is fixed to 0.25 ) on community size-structure. c)

577 Effect of disturbance frequency and d) intensity on average total biomass (in log), for different

578 intensities (c) and frequencies (d), respectively. Points on the black lines in (c) and (d) show the

579 disturbance regimes corresponding to community size-structures of the respective colors displayed

580 in panels (a) and (b).

581

582 Figure 4: Experimental results. a) Effect of disturbance frequency on community size-structure.

583 Vertical bars illustrate mean abundance (individuals/ $\mu$ l) and its standard deviation over 21 time

584 points and 6 replicates for each size-class $(\mu \mathrm{m} 2)$. Disturbance intensity is fixed to $I=90 \%$; other

585 intensities are shown in Fig. S2 and statistics in Table S2. b) Effect of disturbance intensity on

586 community size-structure. Disturbance frequency is fixed to $f=0.33$, other frequencies are

587 shown in Fig. S3 and statistics in Table S2. Controls are in grey (undisturbed environment) and

588 axes are on a logarithmic scale. c) Effect of disturbance frequency on total community biomass

589 (temporal mean, $\mathrm{n}=6$ for treatments, $\mathrm{n}=8$ for controls, in $\mu \mathrm{m} 2 / \mu \mathrm{l}$ ). Disturbance intensity is fixed

590 to $I=90 \%$ as in panel (a); other intensities are shown in Fig. S2. All frequencies have a

591 significant negative effect on total biomass compared to controls: Welch two sample t-tests: $f_{0.08:}$

$592 \mathrm{t}=8, \mathrm{p}$-value $<0.001, f_{0.11} \mathrm{t}=8.5, \mathrm{p}$-value $<0.001, f_{0.16:} \mathrm{t}=13.2, \mathrm{p}$-value $<0.001, f_{0.33:} \mathrm{t}=14.2$,

593 p-value < 0.001. d) Effect of disturbance intensity on total community biomass (temporal mean, $n$

$594=6$ for treatments, $\mathrm{n}=8$ for controls, in $\mu \mathrm{m} 2 / \mu \mathrm{l}$ ). Disturbance frequency is fixed to $f=0.33$ as in

595 panel (b); other frequencies are shown in Fig. S3. All intensities except $I=10 \%$ and $30 \%$ have a

596 significant negative effect on total biomass compared to controls: $I_{10 \%}: \mathrm{t}=0.75$, $\mathrm{p}$-value $=0.48$,

$597 I 30 \%: \mathrm{t}=0.5, \mathrm{p}$-value $=0.63, I_{50 \%}: \mathrm{t}=6.1, \mathrm{p}$-value $<0.001, I 70 \%: \mathrm{t}=12.7, \mathrm{p}$-value $<0.001, I 90 \%: \mathrm{t}=$ 598 14.2, p-value $<0.001$ 
600 Figure 5: Comparison between experimental results and model predictions. a) Predicted vs.

601 observed mean abundance $N$ relative to carrying capacity $K$ in the twelve size-classes for all the 602 disturbance regimes $(\mathrm{n}=240)$. Solid line: linear regression $[\mathrm{y}=-0.012+1.01 \mathrm{x}, \mathrm{R} 2=0.96$, $\mathrm{p}$-value

$603<0.001$. Standard error for slope: 0.01, intercept: 0.02]. Dashed line indicates a 1:1 relationship.

604 b) Predicted effect of disturbance frequency on the community size-structure of experimental 605 communities. Disturbance intensity is fixed to $I=90 \%$; other disturbance regimes are shown in

606 Figs. S4-S5. Controls are in black (undisturbed environment) and axes are on a logarithmic scale.

607 c) Observed effect of disturbance frequency on the community size-structure of experimental

608 communities (similar to Fig. 4a). d) Difference between observed and predicted mean abundance

609 for each size-class.

611 Figure 6: Graphical summary of the effects of disturbances on the shape of size-abundance

612 pyramids. Panels (a) and (b) show size-abundance pyramids for increasing disturbance frequency

613 and intensity, respectively (same analytical results as in Fig. 3a-b). Panel (c) illustrates the expected

614 change in the shape of size-abundance pyramids resulting from a predator-prey dynamic. Lines and

615 points in panel (c) represent isoclines of disturbance regimes $\{I, T\}$ under which we can expect a

616 predation-release effect leading to wider bases of size-abundance pyramids. Points represent the

617 disturbance intensity for which prey species switch from higher to lower mean abundances at

618 dynamical equilibrium in presence compared to in absence of disturbances, for a given disturbance

619 frequency and a set of predator parameters. Black points are estimated for a smaller prey, i.e. with

620 higher growth rate, than grey points (see detailed method in Appendix 3 and Table S4 for parameter 621 values). 
Jacquet et al.

Trophic pyramid

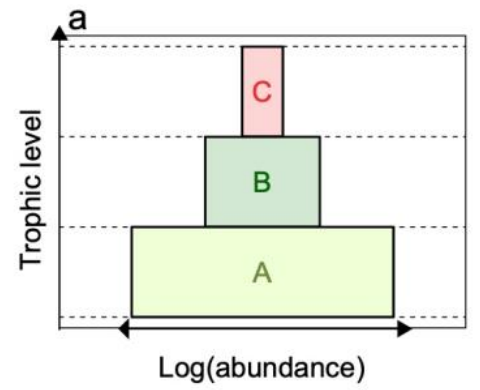

622

623 Figure 1.
How pulse disturbances shape size-abundance pyramids

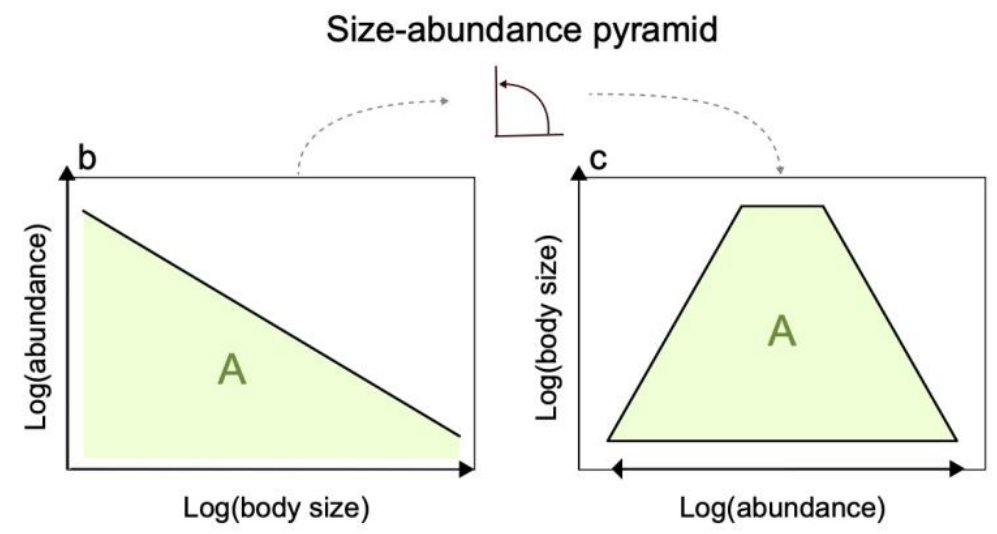



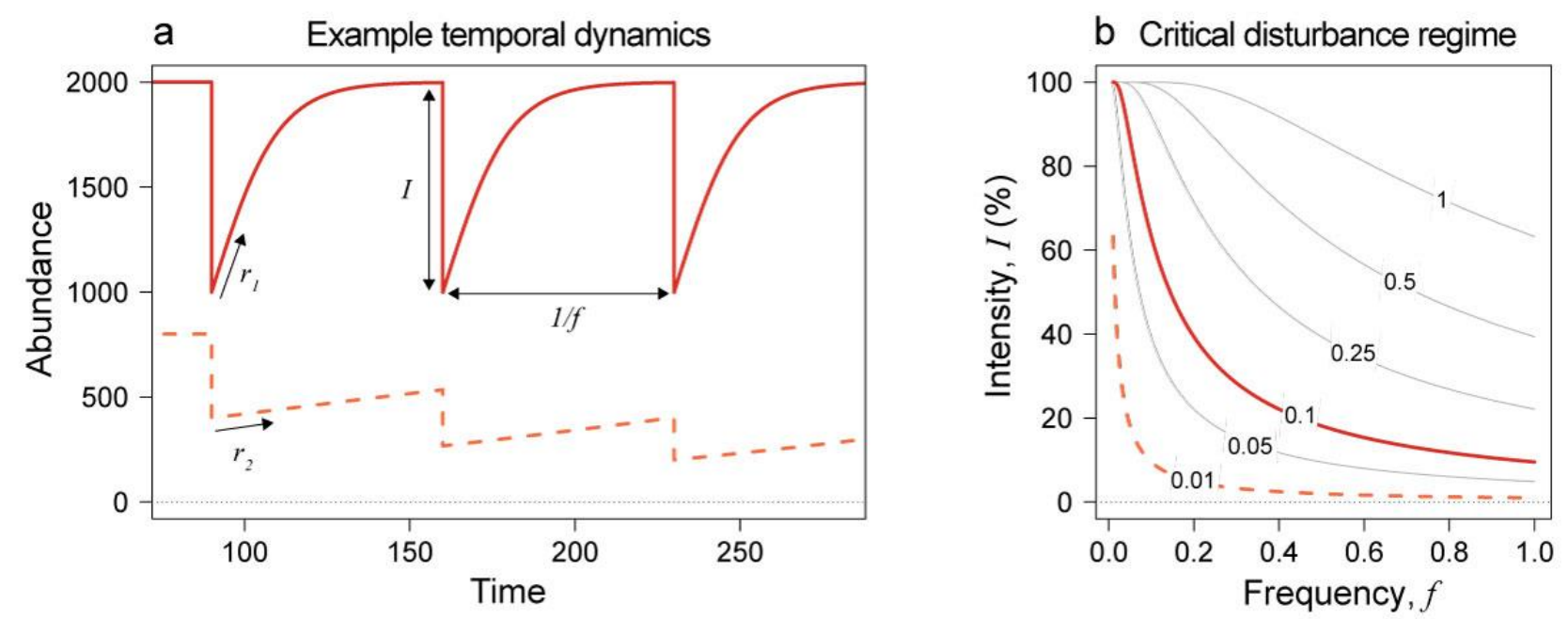

625 Figure 2. 

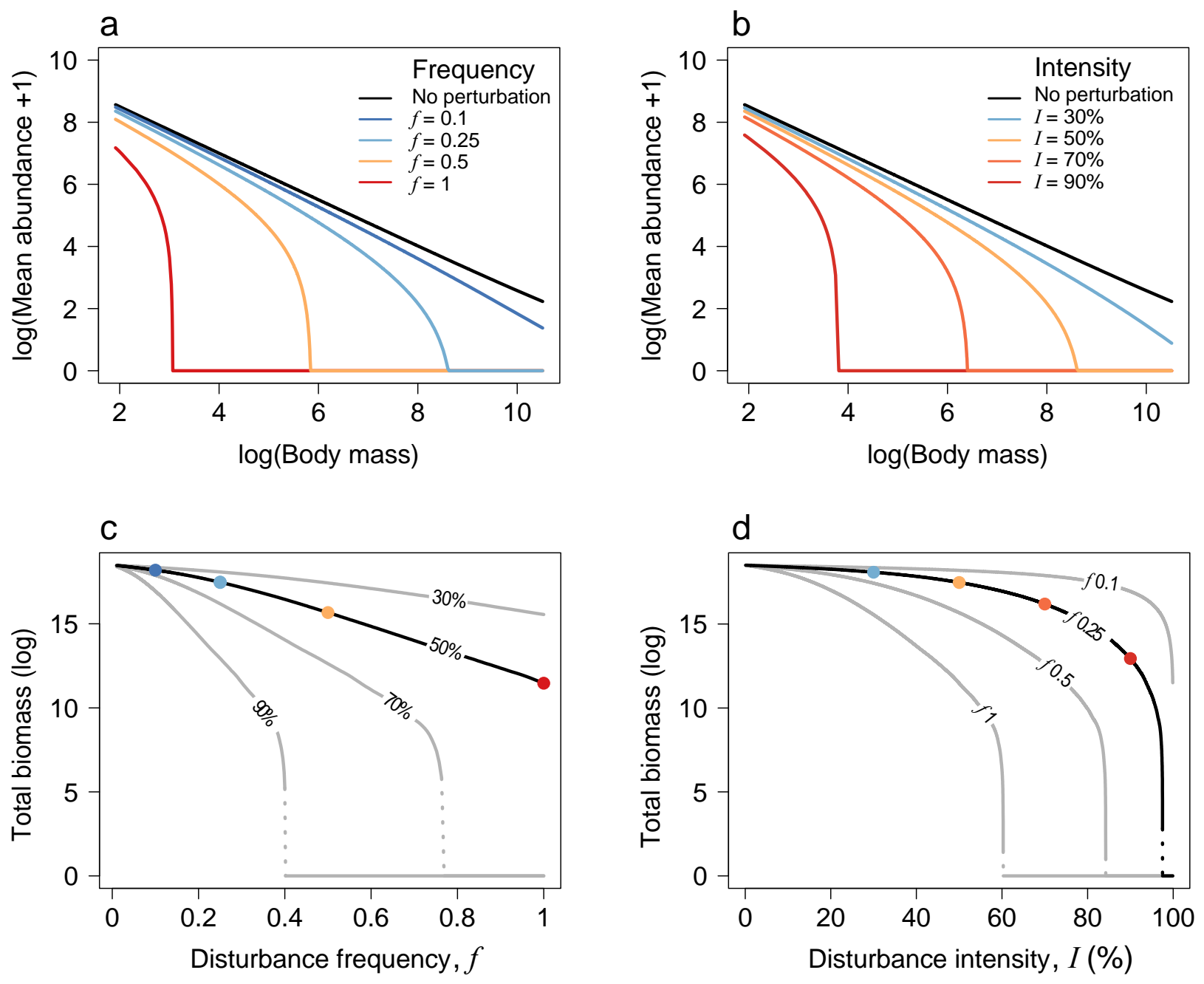

628 Figure 3. 

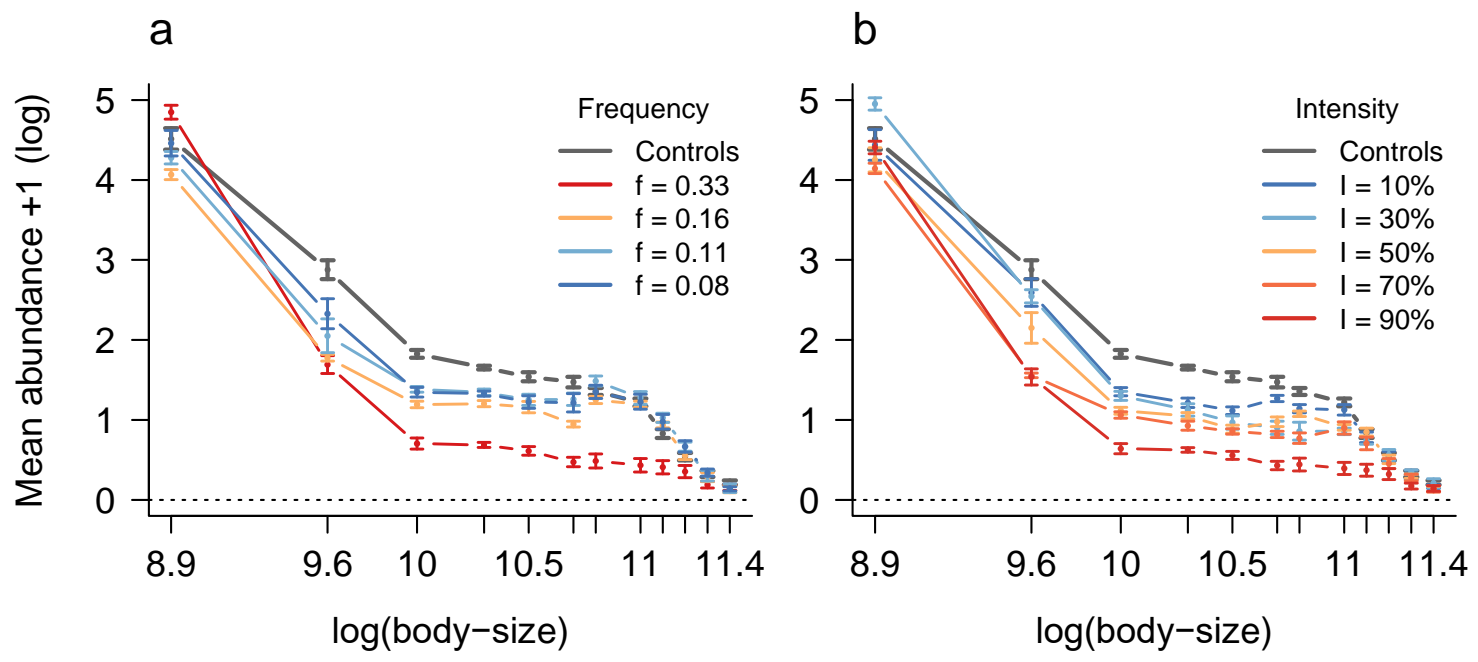

C

d
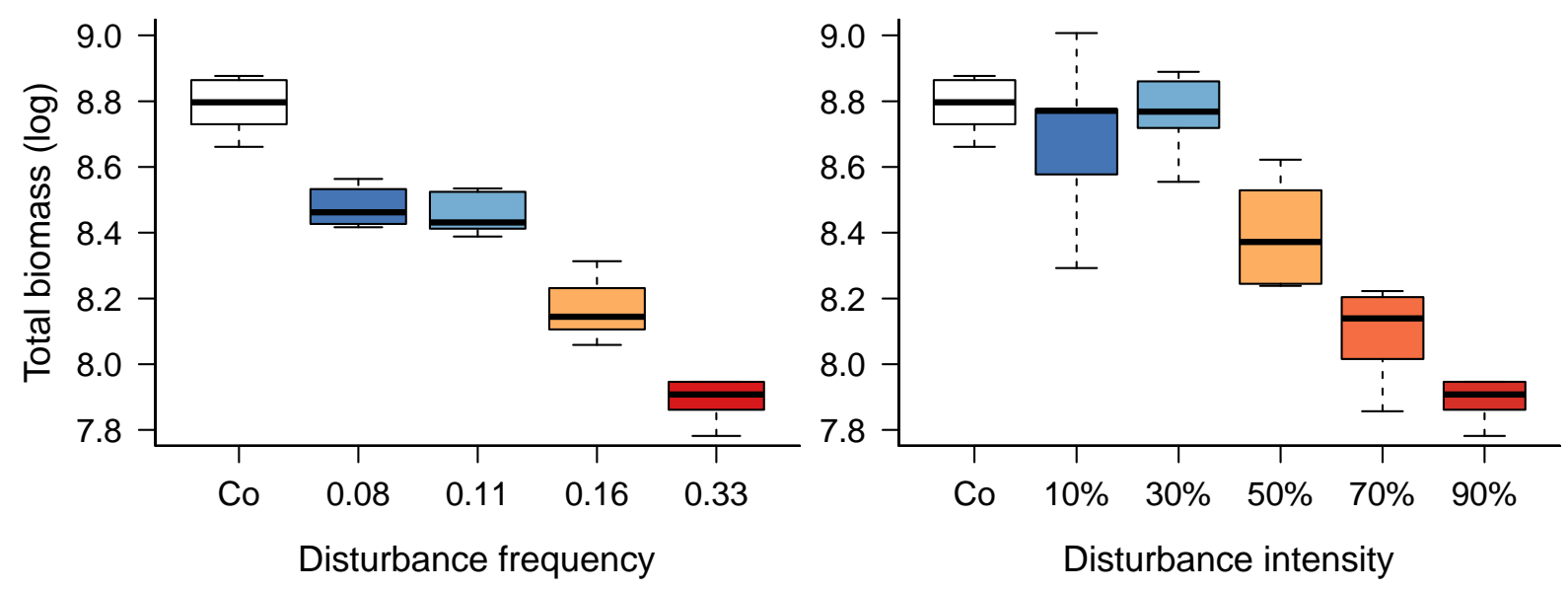

629

$630 \quad$ Figure 4.

631 

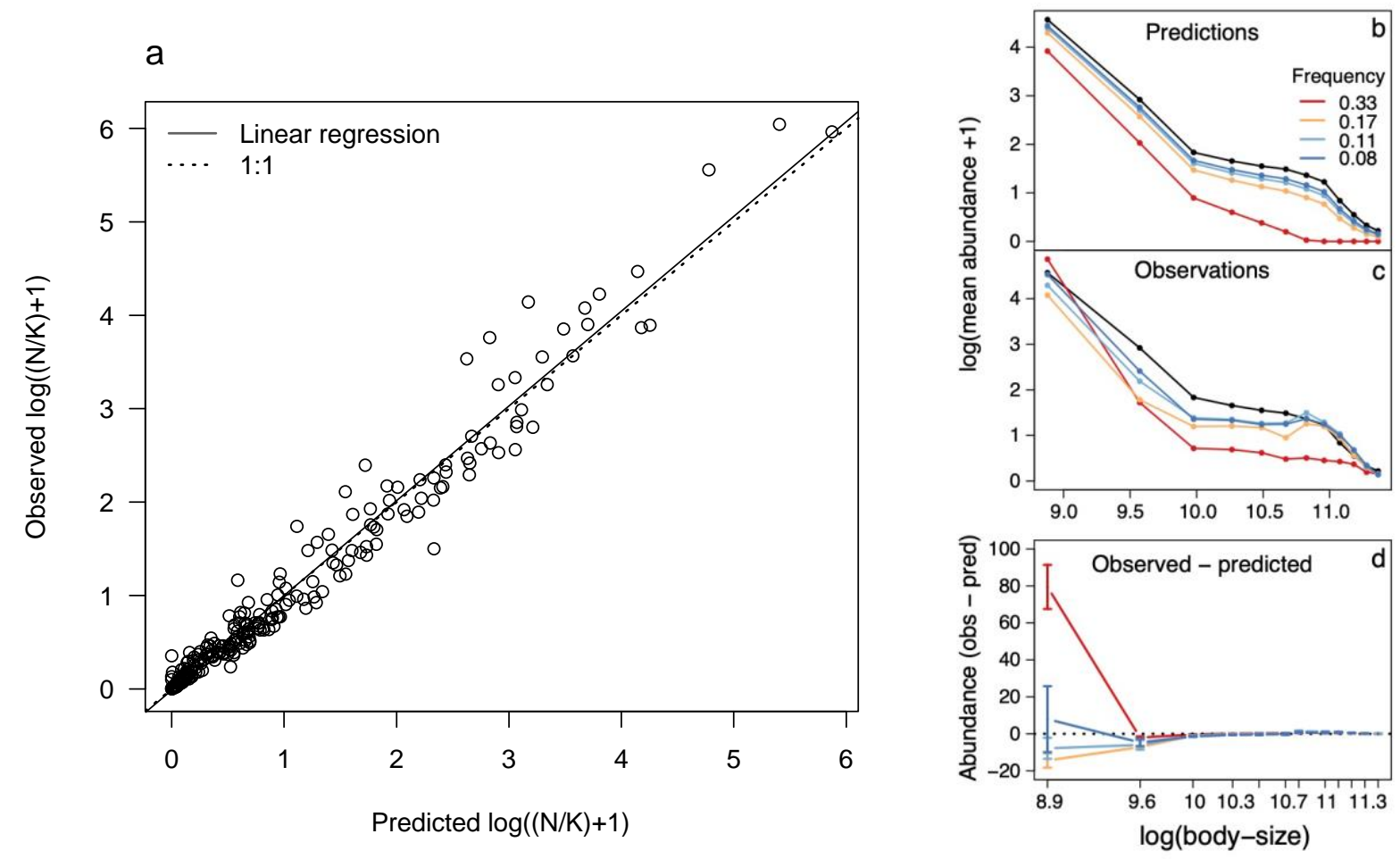

\section{$633 \quad$ Figure 5.}


Jacquet et al.

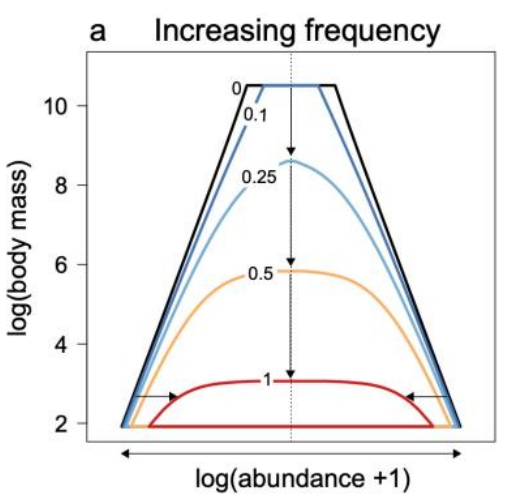

How pulse disturbances shape size-abundance pyramids

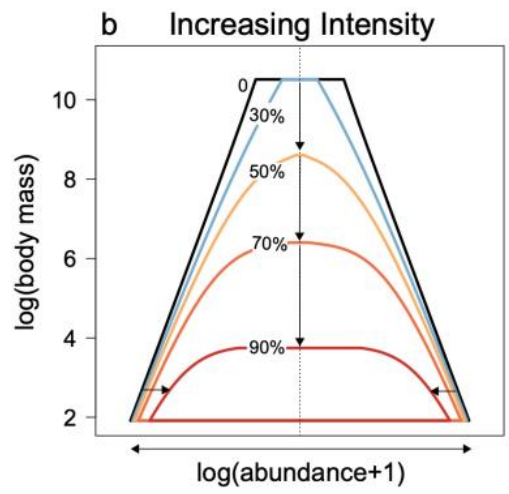

\section{$635 \quad$ Figure 6.}

